\title{
On Concrete Spectral Properties of a Twisted Laplacian Associated with a Central Extension of the Real Heisenberg Group
}

\author{
Aymane El Fardi, Allal Ghanmi, and Ahmed Intissar \\ Center of Mathematical Research and Applications of Rabat (CeReMAR), Analysis and Spectral Geometry (AGS), \\ Laboratory of Mathematical Analysis and Applications (LAMA), Department of Mathematics, Faculty of Sciences, \\ Mohammed V University, P.O. Box 1014, Rabat, Morocco \\ Correspondence should be addressed to Allal Ghanmi; allalghanmi@gmail.com
}

Received 19 April 2017; Revised 13 June 2017; Accepted 27 June 2017; Published 20 August 2017

Academic Editor: Ricardo Weder

Copyright (C) 2017 Aymane El Fardi et al. This is an open access article distributed under the Creative Commons Attribution License, which permits unrestricted use, distribution, and reproduction in any medium, provided the original work is properly cited.

We consider the special magnetic Laplacian given by $\Delta_{v, \mu}=4 \sum_{j=1}^{n}\left(\partial^{2} / \partial z_{j} \partial \overline{z_{j}}\right)+2 i \nu(E+\bar{E}+n)+2 \mu(E-\bar{E})-\left(\nu^{2}+\mu^{2}\right)|z|^{2}$. We show that $\Delta_{\gamma, \mu}$ is connected to the sub-Laplacian of a group of Heisenberg type given by $\mathbb{C} \times{ }_{\omega} \mathbb{C}^{n}$ realized as a central extension of the real Heisenberg group $H_{2 n+1}$. We also discuss invariance properties of $\Delta_{\gamma, \mu}$ and give some of their explicit spectral properties.

\section{Introduction}

The scaled Landau Hamiltonian (special Hermite operator)

$$
H_{\mu}:=4 \sum_{j=1}^{n} \frac{\partial^{2}}{\partial z_{j} \partial \bar{z}_{j}}+2 \mu(E-\bar{E})-\mu^{2}|z|^{2},
$$

where $E$ is the Euler operator and $\bar{E}$ is its complex conjugate, describes the quantum behavior of a charged, spinless particle on the configuration space $\mathbb{C}^{n}$ under the influence of a constant magnetic field. It has been considered and studied from different point of views in physics as in mathematics [1-7]. It goes back to L. D. Landau (for $n=1$ ) and plays an important role in many different contexts such as Feynman path integral (in Feynman-Kac formula), oscillatory stochastic integral, and theory of lattices electrons in uniform magnetic field. See Bellissard [8] and the rich list of references therein.

In the present paper, we study the spectral properties of the second-order differential operator

$$
\begin{aligned}
\Delta_{\nu, \mu} & =4 \sum_{j=1}^{n} \frac{\partial^{2}}{\partial z_{j} \partial \bar{z}_{j}}+2(\mu+i \nu) E-2(\mu-i v) \bar{E} \\
- & \left(\nu^{2}+\mu^{2}\right)|z|^{2}+2 i v n,
\end{aligned}
$$

acting on the free Hilbert space $L^{2}\left(\mathbb{C}^{n}, d m\right)$. The parameters $\nu$ and $\mu$ are assumed to be real such that $\mu>0$. The particular case of $\nu=0$ leads to $\Delta_{0, \mu}=H_{\mu}$. Thus, the Laplacian $\Delta_{\nu, \mu}$ can be seen as the Landau Hamiltonian $H_{\mu}$ perturbed by the first-order differential operator

$$
D_{\nu}:=2 i v(E+\bar{E}+n)-v^{2}|z|^{2} .
$$

In fact we have

$$
\Delta_{\gamma, \mu}=H_{\mu}+D_{v}
$$

Both $\Delta_{\gamma, \mu}$ and $H_{\mu}$ correspond to an isotropic magnetic field of constant strength $\mu$. However, $\Delta_{v, \mu}$ is associated with a specific magnetic vector potential (see (38)) issued from the symmetric gauge by a special gauge involving the parameter $\nu$. This gauge is intimately connected to $D_{\nu}$. Geometrically, as is the case for $H_{\mu}$ (see $\left.[9,10]\right), \Delta_{\nu, \mu}$ represents a Bochner Laplacian on the smooth sections of a Hermitian line bundle with connection over the manifold $M=\mathbb{C}^{n}$.

The real motivation of considering such magnetic quantum Hamiltonian, and therefore the corresponding magnetic potential vector in (38), lies in the study of the space of biweighted holomorphic automorphic functions (defined by 
the means of the projective representation discussed in Section 4). Such space can be considered as the automorphic picture of the classical Bargmann's space of entire functions which has physical implementation. In fact, such phase space is known to be unitary isomorphic to the quantum mechanical configuration space $L^{2}(\mathbb{R} ; d x)$ on the real line (via the so-called Segal-Bargmann transform). One can also realize it as $L^{2}$-eigenspace associated with the lowest Landau level of the Landau Hamiltonian $H_{\mu}$. The same observation holds when dealing with the so-called holomorphic automorphic functions. In advantage, such Landau Hamiltonian leaves invariant such space [11]. Accordingly, the first main object was the introduction of a special magnetic Schrödinger operator satisfying the following conditions:

(i) Leaving invariant the space of biweighted automorphic functions (not necessarily holomorphic).

(ii) The eigenspace associated with the lowest eigenvalue reduces to the space of biweighted holomorphic automorphic functions.

The concrete construction gives rise to the magnetic quantum Hamiltonian $\Delta_{v, \mu}$ given by (2).

The main results to which is aimed this paper concern the realization of $\Delta_{v, \mu}$ as a magnetic Schrödinger operator associated with a specific potential vector (Section 4 ). The connection to the sub-Laplacian of a group of Heisenberg type given by $\mathbb{C} \times{ }_{\omega} \mathbb{C}^{n}$ is also established (see Section 3 ). This group is realized as a central extension of the standard Heisenberg group $H_{2 n+1}=\left(\mathbb{R} \times \mathbb{C}^{n}, \cdot \Im m \omega\right)$. In this new group, the symplectic form is extended and replaced by a Hermitian product (details in Section 2). Invariance properties of $\Delta_{\nu, \mu}$ are discussed in Section 3 and concrete description of its $L^{2}$ spectral analysis is presented in Section 5. More precisely, we show that the spectrum is discrete and independent of the parameter $v$ and coincides with the Landau energy levels of the Landau Hamiltonian $H_{\mu}$ in (1). Moreover, each eigenvalue occurs with infinite multiplicity. An orthogonal basis for every $L^{2}$-eigenspace is next described and the corresponding reproducing kernel is given in a closed form. In Section 6, we use the factorization method [12-16] to generate eigenfunctions of $\Delta_{\gamma, \mu}$ in terms of multivariate version of complex Hermite polynomials. For the case of the twisted Laplacian of the standard Heisenberg group, one can refer to $[6,7,17]$.

\section{The Group $N_{\omega}=\mathbb{C} \times{ }_{\omega} \mathbb{C}^{n}$ as a Central Extension of the Heisenberg Group $H_{2 n+1}=\mathbb{R} \times_{\Im m \omega} \mathbb{C}^{n}$}

In this section, we follow the exposition given in [18] in order to realize $N_{\omega}:=\mathbb{C} \times{ }_{\omega} \mathbb{C}^{n}$ as a central extension of the Heisenberg group $H_{2 n+1}:=\mathbb{R} \times_{\Im m \omega} \mathbb{C}^{n}$, where $\omega(z, w)$ denotes the standard Hermitian form on $\mathbb{C}^{n}$. Indeed, if $(K, \bullet)$ and $(G, \odot)$ are two abelian groups and $\psi: K \times K \rightarrow G$ is a given mapping, then we define on $G \times K$ the ${ }_{\psi}{ }$-law by

$$
\left(z_{0} ; z\right){ }_{\psi}\left(w_{0} ; w\right)=\left(z_{0} \odot w_{0} \odot \psi(z, w) ; z \bullet w\right) .
$$

We say that $G \times{ }_{\psi} K$ is a central extension of $(K, \bullet)$ by $(G, \odot)$ associated with $\psi$ if the short sequence

$$
0 \longrightarrow K \longrightarrow G \times_{\psi} K \longrightarrow G \longrightarrow 0
$$

is exact and such that $K$ is in $Z(G)$, the center of the group $E$. This holds if one of the following two equivalent assertions is satisfied:

(i) $\psi$ preserves the neutral element $\psi\left(0_{K}, 0_{K}\right)=0_{G}$ and verifies the cocycle relation

$$
\psi(x, y) \odot \psi(x \bullet y, z)=\psi(x, y \bullet z) \odot \psi(y, z) .
$$

For every $x, y, z \in K$.

(ii) $G \times{ }_{\psi} K:=\left(G \times K,{ }_{\psi}\right)$ is a group.

Now, let $\mathbb{R}^{2}=\mathbb{R}_{s} \times \mathbb{R}_{t}$ be the real $(s, t)$-plane identified with the complex plane $\mathbb{C}=\left\{z_{0}=s+i t ; s, t \in \mathbb{R}\right\}$ and $\mathbb{C}^{n}$ denotes the complex $n$-space endowed with its standard Hermitian form

$$
\omega(z, w):=\langle z, w\rangle=\sum_{j=1}^{n} z_{j} \bar{w}_{j}
$$

for $z=\left(z_{1}, z_{2}, \ldots, z_{n}\right)$ and $w=\left(w_{1}, w_{2}, \ldots, w_{n}\right)$ in $\mathbb{C}^{n}$. We define $N_{\omega}=\mathbb{C} \times{ }_{\omega} \mathbb{C}^{n}$ to be the set $\mathbb{C} \times \mathbb{C}^{n}$ endowed with the ${ } \omega^{\text {-law given by }}$

$$
\left(z_{0} ; z\right) \cdot{ }_{\omega}\left(w_{0} ; w\right)=\left(z_{0}+w_{0}+\langle z, w\rangle ; z+w\right) .
$$

Under (9), $N_{\omega}:=\mathbb{C} \times{ }_{\omega} \mathbb{C}^{n}$ is a noncommutative nilpotent group of step two with center $Z\left(N_{\omega}\right)=\mathbb{C} \times{ }_{\omega}\{0\}=(\mathbb{R} \times$ $\mathbb{R}) \times{ }_{\omega}\{0\}$. The identity element is $(0 ; 0)$ and the symmetric element of given $\left(z_{0} ; z\right)$ is $\left(-z_{0}-\langle z, z\rangle ;-z\right)$. Notice for instance that the ${ }_{\omega}$-law given by (9) can be rewritten in the coordinates $z_{0}=(s, t), w_{0}=\left(s^{\prime}, t^{\prime}\right)$, and $z, w \in \mathbb{C}^{n}$ as follows:

$$
\begin{aligned}
& ((s, t) ; z) \cdot{ }_{\omega}\left(\left(s^{\prime}, t^{\prime}\right) ; w\right) \\
& =\left(\left(s+s^{\prime}+\operatorname{Re}\langle z, w\rangle, t+t^{\prime}+\Im m\langle z, w\rangle\right) ; z+w\right) .
\end{aligned}
$$

Hence, endowing the set $\mathbb{R}_{t} \times \mathbb{C}^{n}$ with the $\cdot \Im m \omega$-law, given by

$$
(t ; z) \cdot \Im m \omega\left(t^{\prime} ; w\right)=\left(t+t^{\prime}+\Im m\langle z, w\rangle ; z+w\right),
$$

makes $\mathbb{R} \times_{\Im m \omega} \mathbb{C}^{n}$ a group, which is nothing else than the classical real Heisenberg group of dimension $2 n+1$. One can notice easily that $\left(\mathbb{C} \times \mathbb{C}^{n}, \cdot{ }_{\omega}\right)$, in addition of being the central extension of $\mathbb{C}^{n}$ by $\mathbb{C}$ associated with the map $\psi=\omega$, can also be viewed, due to (10), as the central extension of $\left(\mathbb{R}_{t} \times \mathbb{C}^{n}, \cdot \Im m \omega\right)$ by $\mathbb{R}_{s}$ associated with $\psi=\Re e \omega$. This can be stated otherwise using directly the following definition: the projection mapping $q(s, t ; z)=(t ; z)$, from $\left(\mathbb{R}_{s} \times \mathbb{R}_{t}\right) \times_{\omega} \mathbb{C}^{n}$ onto $\mathbb{R}_{t} \times_{\Im m \omega} \mathbb{C}^{n}$, is a homomorphism from the group $N_{\omega}=$ $\mathbb{C} \times{ }_{\omega} \mathbb{C}^{n}$ onto the Heisenberg group $H_{2 n+1}=\mathbb{R} \times_{\Im m \omega} \mathbb{C}^{n}$. Moreover, the kernel of $q$ is given by

$$
\operatorname{ker} q=\{(s, 0 ; 0), s \in \mathbb{R}\}=\left(\mathbb{R}_{s} \times\{0\}\right) \times_{\omega}\{0\}
$$

and is clearly contained in the center $Z\left(N_{\omega}\right)$. Thus, we may say that the group $N_{\omega}=\mathbb{C} \times{ }_{\omega} \mathbb{C}^{n}$ is a central extension of the Heisenberg group $H_{2 n+1}=\mathbb{R} \times_{\Im m \omega} \mathbb{C}^{n}$ by $\left(\mathbb{R}_{s},+\right)$; that is, we have $\left(\mathbb{C} \times{ }_{\omega} \mathbb{C}^{n}\right) / \operatorname{ker} q=\left(\mathbb{C} \times{ }_{\omega} \mathbb{C}^{n} / \mathbb{R}_{s}\right)=H_{2 n+1}$. Accordingly, harmonic analysis on our group $N_{\omega}=\mathbb{C} \times_{\omega} \mathbb{C}^{n}$ will have many links to that on the classical Heisenberg group. 


\section{Explicit Formula for the Sub-Laplacian $\mathscr{L}_{\omega}$ on $N_{\omega}=\mathbb{C} \times{ }_{\omega} \mathbb{C}^{n}$}

The group $N_{\omega}=\mathbb{C} \times{ }_{\omega} \mathbb{C}^{n}$ with the ${ }_{\omega}$-law given in (9) is a real Lie group of dimension $2 n+2$, and its tangent space at its neutral element is given by $T_{(0 ; 0)} N_{\omega}=(\mathbb{C},+) \times\left(\mathbb{C}^{n},+\right)$ viewed as a real vector space of dimension $2 n+2$. In fact, $N_{\omega}$ is naturally equipped with the standard differentiable structure on Euclidean spaces, generated by the coordinates system $\left\{\left(\mathbb{C} \times \mathbb{C}^{n}, x\right)\right\}$, where $x$ is the coordinates map

$$
\begin{aligned}
x: \mathbb{C} \times \mathbb{C}^{n} & \longrightarrow \mathbb{R}^{2 n+2} ; \\
\left(z_{0} ; z\right) & \longmapsto\left(s, t ; x_{1}, y_{1}, x_{2}, y_{2}, \ldots, x_{n}, y_{n}\right) .
\end{aligned}
$$

The group action and the group symmetric maps are smooth under this differentiable structure. Denote by $\mathfrak{n}_{\omega}$ its associated Lie algebra composed of all left-invariant vector fields on $N_{\omega}$ and endowed with the standard bracket on vector fields. For the sake of giving the explicit formula for the subLaplacian $\mathscr{L}_{\omega}$ on $N_{\omega}=\mathbb{C} \times_{\omega} \mathbb{C}^{n}$, a basis of $\mathfrak{n}_{\omega}$ formed by first-order differential operators on functions of $N_{\omega}$ is needed. Define the left action by a fixed element $\left(z_{0} ; z\right) \in N_{\omega}$ by

$$
\begin{aligned}
\ell_{\left(z_{0} ; z\right)}: N_{\omega} & \longrightarrow N_{\omega} ; \\
\left(w_{0} ; w\right) & \longmapsto\left(z_{0} ; z\right) \cdot_{\omega}\left(w_{0} ; w\right) .
\end{aligned}
$$

This map is a diffeomorphism with respect to the Lie group structure. Hence, it is possible to extend its push-forward to act on vector fields. Furthermore, its action on a vector field $X$ is given explicitly by

$$
\ell_{g_{*}} X_{p} f=X_{p}\left(f \circ \ell_{g}\right)
$$

for test data $p \in N_{\omega}$ and $f$ a smooth function of $N_{\omega}$. By definition, a vector field $X$ is said to be left-invariant if the equality $\ell_{g_{*}} X=X$ holds.

In order to construct a left-invariant vector field basis, we take a basis of the tangent vectors at the identity and generate from each vector of the tangent basis a left-invariant vector field by pushing it forward using $\ell_{\left(z_{0}, z\right)}$. Recall that a basis of the tangent vector space $T_{(0 ; 0)} N_{\omega}$ acting on smooth functions $f$ is given by

$$
\begin{aligned}
\left(\frac{\partial}{\partial x^{i}}\right)_{(0 ; 0)} f:=\left.\partial_{i}\left(f \circ x^{-1}\right)\right|_{x(0,0)} & \\
& i=1,2, \ldots, 2 n+2,
\end{aligned}
$$

where $\partial_{i}$ is the ordinary partial derivative with respect to the $i$ th variable. We can now carry out the following computation in order to find generators for $\mathfrak{n}_{\omega}$ :

$$
\begin{aligned}
\ell_{\left(z_{0} ; z\right)}\left(\frac{\partial}{\partial x^{i}}\right)_{(0 ; 0)} f & =\left(\frac{\partial}{\partial x^{i}}\right)_{(0 ; 0)}\left(f \circ \ell_{\left(z_{0} ; z\right)}\right) \\
& =\left.\partial_{i}\left(\left(f \circ \ell_{\left(z_{0}, z\right)}\right) \circ x^{-1}\right)\right|_{x(0,0)} .
\end{aligned}
$$

We plug in $x^{-1} \circ x$ in the middle of the last equation and we use the multivariable chain rule to get

$$
\begin{aligned}
& \ell_{\left(z_{0} ; z\right) *}\left(\frac{\partial}{\partial x^{i}}\right)_{(0 ; 0)} f=\left.\partial_{i}\left(\left(f \circ x^{-1}\right) \circ\left(x \circ \ell_{\left(z_{0} ; z\right)} \circ x^{-1}\right)\right)\right|_{x(0,0)} \\
& =\left.\sum_{m=1}^{2 n+2} \partial_{m}\left(f \circ x^{-1}\right)\right|_{x \circ \ell_{\left(z_{0} ;\right)^{\circ}} x^{-1} \circ x(0,0)} \times\left.\partial_{i}\left(x^{m} \circ \ell_{\left(z_{0} ; z\right)} \circ x^{-1}\right)\right|_{x(0,0)} \\
& =\sum_{m=1}^{2 n+2} \mathbf{J}_{m, i} \times\left(\frac{\partial}{\partial x^{m}}\right)_{\left(z_{0} ; z\right)},
\end{aligned}
$$

where $\mathbf{J}_{m, i}:=\left.\partial_{i}\left(x^{m} \circ \ell_{\left(z_{0} ; z\right)} \circ x^{-1}\right)\right|_{x(0,0)}$ and $x^{m} \circ \ell_{\left(z_{0} ; z\right)} \circ x^{-1}$ is the $m$ th coordinate map of $x \circ \ell_{\left(z_{0} ; z\right)} \circ x^{-1}$. Explicitly, we have

$$
\begin{aligned}
x & \circ \ell_{\left(z_{0} ; z\right)} \circ x^{-1}\left(s^{\prime}, t^{\prime}, x_{1}^{\prime}, y_{1}^{\prime}, \ldots, x_{n}^{\prime}, y_{n}^{\prime}\right)=\left(s+s^{\prime}\right. \\
& +\sum_{j=1}^{n}\left(x_{j} x_{j}^{\prime}+y_{j} y_{j}^{\prime}\right), t+t^{\prime}+\sum_{j=1}^{n}\left(y_{j} x_{j}^{\prime}-x_{j} y_{j}^{\prime}\right), x_{1} \\
& \left.+x_{1}^{\prime}, y_{1}+y_{1}^{\prime}, \ldots, y_{n}+y_{n}^{\prime}\right) .
\end{aligned}
$$

Therefore, the $\mathbf{J}_{m, i}$ can be viewed as the components of the following Jacobian matrix:

$$
\mathbf{J}:=\mathbf{J}_{(0, \ldots, 0)}=\left(\begin{array}{ccccccc}
1 & 0 & x_{1} & y_{1} & \cdots & x_{n} & y_{n} \\
0 & 1 & y_{1} & -x_{1} & \cdots & y_{n} & -x_{n} \\
\vdots & 0 & \ddots & & & & 0 \\
\vdots & & \ddots & & & \\
\vdots & & & \ddots & & \\
\vdots & & & & & \ddots & \\
0 & 0 & \cdots & & & \cdots & 1
\end{array}\right) .
$$

Reading vertically, column by column, we find the following basis:

$$
\begin{aligned}
S & =\left(\frac{\partial}{\partial s}\right), \\
T & =\left(\frac{\partial}{\partial t}\right), \\
X_{j} & =x_{j}\left(\frac{\partial}{\partial s}\right)+y_{j}\left(\frac{\partial}{\partial t}\right)+\left(\frac{\partial}{\partial x_{j}}\right), \\
Y_{j} & =y_{j}\left(\frac{\partial}{\partial s}\right)-x_{j}\left(\frac{\partial}{\partial t}\right)+\left(\frac{\partial}{\partial y_{j}}\right) .
\end{aligned}
$$


Note that we are using the coordinates $z_{0}=s+i$ and $z_{j}=$ $x_{j}+i y_{j}$ with

$$
\begin{gathered}
\frac{\partial}{\partial x^{1}}=\frac{\partial}{\partial s}, \\
\frac{\partial}{\partial x^{2}}=\frac{\partial}{\partial t}, \\
\frac{\partial}{\partial x^{2 j+1}}=\frac{\partial}{\partial x_{j}}, \\
\frac{\partial}{\partial x^{2 j+2}}=\frac{\partial}{\partial y_{j}},
\end{gathered}
$$

for $j=1, \ldots, n$. We summarize the above discussion on $N_{\omega}=$ $\mathbb{C} \times{ }_{\omega} \mathbb{C}^{n}$ and its associated Lie algebra $\mathfrak{n}_{\omega}$ in the following statement.

Proposition 1. The real vector fields

$$
\begin{aligned}
& S=\frac{\partial}{\partial s}, \\
& T=\frac{\partial}{\partial t}
\end{aligned}
$$

together with $X_{j}, Y_{j} ; j=1, \ldots, n$ given by

$$
\begin{aligned}
& X_{j}=x_{j}\left(\frac{\partial}{\partial s}\right)+y_{j}\left(\frac{\partial}{\partial t}\right)+\left(\frac{\partial}{\partial x_{j}}\right), \\
& Y_{j}=y_{j}\left(\frac{\partial}{\partial s}\right)-x_{j}\left(\frac{\partial}{\partial t}\right)+\left(\frac{\partial}{\partial y_{j}}\right)
\end{aligned}
$$

form a basis for $\mathfrak{n}_{\omega}$. Moreover, they satisfy the following commutation relations of Heisenberg type:

$$
\begin{aligned}
{\left[S, X_{j}\right] } & =\left[S, Y_{j}\right]=0, \\
{\left[T, X_{j}\right] } & =\left[T, Y_{j}\right]=0, \\
{[S, T] } & =0, \\
{\left[X_{j}, X_{k}\right] } & =\left[Y_{j}, Y_{k}\right]=0, \\
{\left[X_{j}, Y_{k}\right] } & =-2 \delta_{j k} T
\end{aligned}
$$

for all $j, k=1, \ldots, n$.

Remark 2. As expected we see, in view of the above proposition, that the Lie algebra $\mathfrak{n}_{\omega}$ of $N_{\omega}=\mathbb{C} \times{ }_{\omega} \mathbb{C}^{n}$ with $\omega(z, w)=$ $\langle z, w\rangle$ is also a central extension of the classical Heisenberg algebra $H_{2 n+1}=\mathbb{R} \times_{\Im m \omega} \mathbb{C}^{n}$ generated by the vector fields

$$
\begin{array}{r}
\left\{T=\frac{\partial}{\partial t}, \widetilde{X}_{j}=-y_{j} \frac{\partial}{\partial t}+\frac{\partial}{\partial x_{j}}, \tilde{Y}_{j}=x_{j} \frac{\partial}{\partial t}+\frac{\partial}{\partial y_{j}}\right\} \\
j=1, \ldots, n
\end{array}
$$

with the nontrivial commutation relation $\left[\widetilde{X}_{j}, \widetilde{Y}_{k}\right]=-2 T$, where $\left(x_{j}, y_{j}\right) ; j=1, \ldots, n$, are the coordinates of $\mathbb{C}^{n}=\mathbb{R}^{2 n}$.
Remark 3. To build such left-invariant vector fields, one can also look for a one parameter group of $N_{\omega}$, that is, a group homomorphism $\gamma:(\mathbb{R},+) \rightarrow N_{\omega}$ satisfying

$$
\dot{\gamma}(0)=\left.\frac{d \gamma}{d \varepsilon}(\varepsilon)\right|_{\varepsilon=0}=\left(v_{0} ; v\right) \in T_{(0 ; 0)} N_{\omega}=\mathbb{C} \times \mathbb{C}^{n} .
$$

According to the above discussion, we can introduce the following definition of sub-Laplacian on $N_{\omega}=\mathbb{C} \times{ }_{\omega} \mathbb{C}^{n}$.

Definition 4. Let $X_{j}, Y_{j} ; j=1, \ldots, n$, be the vector fields given in Proposition 1. Then, the operator

$$
\mathscr{L}_{\omega}=\sum_{j=1}^{n} X_{j}^{2}+Y_{j}^{2}
$$

is called a sub-Laplacian of $N_{\omega}=\mathbb{C} \times{ }_{\omega} \mathbb{C}^{n}$.

The following proposition gives the explicit differential expression of $\mathscr{L}_{\omega}$ in terms of the Laplace-Beltarmi $\Delta_{\mathbb{R}^{2 n}}$ of $\mathbb{C}^{n}=\mathbb{R}^{2 n}$

$$
\Delta_{\mathbb{R}^{2 n}}:=\sum_{j=1}^{n} \frac{\partial^{2}}{\partial x_{j}^{2}}+\frac{\partial^{2}}{\partial y_{j}^{2}}
$$

and the first-order differential operators $E_{x, y}$ and $F_{x, y}$ defined by

$$
\begin{aligned}
& E_{x, y}:=\sum_{j=1}^{n} x_{j} \frac{\partial}{\partial x_{j}}+y_{j} \frac{\partial}{\partial y_{j}}, \\
& F_{x, y}:=\sum_{j=1}^{n} x_{j} \frac{\partial}{\partial y_{j}}-y_{j} \frac{\partial}{\partial x_{j}} .
\end{aligned}
$$

Namely, we have the following result.

Proposition 5. The sub-Laplacian $\mathscr{L}_{\omega}$ prescribed in Definition 4 is given explicitly in the coordinates $t, s, x_{j}, y_{j}, j=$ $1, \ldots, n$, of $N_{\omega}=\mathbb{C} \times{ }_{\omega} \mathbb{C}^{n}$ as follows:

$$
\begin{aligned}
\mathscr{L}_{\omega}= & \Delta_{\mathbb{R}^{2 n}}+2\left(E_{x, y}+n\right) \frac{\partial}{\partial s}-2 F_{x, y} \frac{\partial}{\partial t} \\
& +\left(|x|^{2}+|y|^{2}\right)\left(\frac{\partial^{2}}{\partial s^{2}}+\frac{\partial^{2}}{\partial t^{2}}\right),
\end{aligned}
$$

where $|x|^{2}=\sum_{j=1}^{n} x_{j}^{2}$ and $|y|^{2}=\sum_{j=1}^{n} y_{j}^{2}$.

Proof. The explicit expression of $\mathscr{L}_{\omega}$ given in Proposition 5 can be handled by straightforward computations.

Remark 6. If we consider the coordinates $(s, t) \in \mathbb{R}^{2}=\mathbb{C}$ and $z=\left(z_{1}, \ldots, z_{n}\right) \in \mathbb{C}^{n}$ with $z_{j}=x_{j}+i y_{j}$, then the subLaplacian $\mathscr{L}_{\omega}$ in (31) can be rewritten as

$$
\begin{gathered}
\mathscr{L}_{\omega}=4 \sum_{j=1}^{n} \frac{\partial^{2}}{\partial z_{j} \partial \overline{z_{j}}}+2(E+\bar{E}+n) \frac{\partial}{\partial s} \\
-2 i(E-\bar{E}) \frac{\partial}{\partial t}+|z|^{2}\left(\frac{\partial^{2}}{\partial s^{2}}+\frac{\partial^{2}}{\partial t^{2}}\right),
\end{gathered}
$$


where $E=\sum_{j=1}^{n} z_{j}\left(\partial / \partial z_{j}\right)$ is the complex Euler operator and $\bar{E}=\sum_{j=1}^{n} \overline{z_{j}}\left(\partial / \partial \overline{z_{j}}\right)$ is its complex conjugate.

Remark 7. The action of $\mathscr{L}_{\omega}$ on functions $F(t ; z)$ on $N_{\omega}=$ $\mathbb{C} \times{ }_{\omega} \mathbb{C}^{n}$ that are independent of the argument $s$ reduces to that of the sub-Laplacian

$$
\widetilde{\mathscr{L}}_{\Im m \omega}=4 \sum_{j=1}^{n} \frac{\partial^{2}}{\partial z_{j} \partial \overline{z_{j}}}-2 i(E-\bar{E}) \frac{\partial}{\partial t}+|z|^{2} \frac{\partial^{2}}{\partial t^{2}}
$$

of the classical Heisenberg group $\mathbb{R} \times_{\mathfrak{\Im} m \omega} \mathbb{C}^{n}=H_{2 n+1}$.

We conclude this section by mentioning that both operators $\mathscr{L}_{\omega}$ and $\widetilde{\mathscr{L}}_{\Im m \omega}$ are not elliptic. But they share many aspects and nice properties of their spectral theory with elliptic operators. We will precise this by giving the concrete description of the spectral eigenfunction problem of the associated elliptic differential operator

$$
\begin{gathered}
\Delta_{\nu, \mu}=4 \sum_{j=1}^{n} \frac{\partial^{2}}{\partial z_{j} \partial \overline{z_{j}}}+2 \mu(E-\bar{E})+2 i v(E+\bar{E}+n) \\
-\left(\nu^{2}+\mu^{2}\right)|z|^{2}=4 \sum_{j=1}^{n} \frac{\partial^{2}}{\partial z_{j} \partial \overline{z_{j}}}+2(\mu+i v) E \\
-2(\mu-i v) \bar{E}-\left(\nu^{2}+\mu^{2}\right)|z|^{2}+2 i v n .
\end{gathered}
$$

Formally, $\Delta_{\nu, \mu}$ is related to $\mathscr{L}_{\omega}$ using partial Fourier transform in $(s, t)$ with $(i \nu, i \mu)$ as dual arguments.

In the next section, we will prove that the operator $\Delta_{v, \mu}$ can also be regarded as a Schrödinger operator in the presence of a uniform magnetic field $\vec{B}_{\mu}=i d \theta_{\nu, \mu}$ on $\mathbb{C}^{n}=\mathbb{R}^{2 n}$ associated with a specific differential 1-form $\theta_{\nu, \mu}$.

\section{Realization of $\Delta_{\gamma, \mu}$ as a Magnetic Schrödinger Operator and Invariance Property}

A magnetic Schrödinger operator on a complete oriented Riemannian manifold $(M, g)$ on scalar functions is in general of the form

$$
H_{\theta}=(d+\operatorname{ext} \theta)^{*}(d+\operatorname{ext} \theta)
$$

where $\theta$ is a given $\mathscr{C}^{1}$ real differential 1 -form on $M$ (magnetic vector potential). Here $d$ stands for the usual exterior derivative acting on the space of differential $p$-forms $\Omega^{p}(M)$, $\operatorname{ext} \theta$ is the operator of exterior left multiplication by $\theta$, that is, $(\operatorname{ext} \theta) \omega=\theta \wedge \omega$, and $(d+\operatorname{ext} \theta)^{*}$ is the formal adjoint of $d+\operatorname{ext} \theta$ with respect to the Hermitian product on $\Omega^{p}=$ $\Omega^{p}(M)$

$$
\langle\alpha, \beta\rangle_{\Omega^{p}}=\int_{M} \alpha \wedge \star \beta
$$

induced by the metric $g$, where $\star$ denotes the Hodge star operator associated with the volume form. From general theory of Schrödinger operators on noncompact manifold
$M$ (see for example [19]), it is known that the operator $H_{\theta}$, viewed as an unbounded operator in $L^{2}(M ; d m)$, is essentially self-adjoint for any smooth measure $d m$.

In our framework $M$ is the complex $n$-space $\mathbb{C}^{n}$ equipped with its Kähler metric

$$
d s^{2}=-\frac{i}{2} \sum_{j=1}^{n} d z_{j} \otimes d \bar{z}_{j}=\sum_{j=1}^{n} d x_{j} \otimes d y_{j}
$$

and the corresponding volume form is $\operatorname{Vol}(z)=d x_{1} d y_{1} \cdots$ $d x_{n} d y_{n}$. Associated with the parameters $\nu$ and $\mu$, we consider the potential vector

$$
\theta_{\nu, \mu}(z):=-\frac{\mu-i \nu}{2} \sum_{j=1}^{n} \overline{z_{j}} d z_{j}+\frac{\mu+i \nu}{2} \sum_{j=1}^{n} z_{j} d \overline{z_{j}} .
$$

Some comments on such vector potential are collected in Remarks 12,13, and 17 below. Thus, we can prove the following result concerning the twisted Laplacian defined by (34).

Proposition 8. For every complex-valued $\mathscr{C}^{\infty}$ function $f$ on $\mathbb{C}^{n}$, we have

$$
\Delta_{\nu, \mu} f=-H_{\theta_{\nu, \mu}} f=-\left(d+\operatorname{ext} \theta_{\nu, \mu}\right)^{*}\left(d+\operatorname{ext} \theta_{\nu, \mu}\right) f .
$$

Proof (sketched). We start by writing $H_{\theta_{\nu, \mu}}:=\left(d+\operatorname{ext} \theta_{\nu, \mu}\right)^{*}(d+$ $\left.\operatorname{ext} \theta_{\nu, \mu}\right)$ as

$$
\begin{aligned}
H_{\theta_{\nu, \mu}}= & d^{*} d f+d^{*} \operatorname{ext} \theta_{\nu, \mu} f+\left(\operatorname{ext} \theta_{\nu, \mu}\right)^{*} d f \\
& +\left(\operatorname{ext} \theta_{\nu, \mu}\right)^{*} \operatorname{ext} \theta_{\nu, \mu} .
\end{aligned}
$$

Next, using the well-known facts $d^{*}=-\star d \star$ and $(\operatorname{ext} \theta)^{*}=$ $\star \operatorname{ext} \theta \star$, we establish the following

$$
\begin{aligned}
& d^{*} d=-4 \sum_{j=1}^{n} \frac{\partial}{\partial z_{j}} \frac{\partial}{\partial \overline{z_{j}}}, \\
& d^{*} \operatorname{ext} \theta_{\nu, \mu} f+\left(\operatorname{ext} \theta_{\nu, \mu}\right)^{*} d \\
& \quad=\sum_{j=1}^{n}\left(-2(\mu+i \nu) z_{j} \frac{\partial}{\partial z_{j}}+2(\mu-i \nu) \overline{z_{j}} \frac{\partial}{\partial \overline{z_{j}}}-2 i \nu\right), \\
& \left(\operatorname{ext} \theta_{\nu, \mu}\right)^{*} \operatorname{ext} \theta_{\nu, \mu}=\left(\mu^{2}+\nu^{2}\right)|z|^{2} .
\end{aligned}
$$

One of the advantages of the realization of $\Delta_{\nu, \mu}$ as (39), with the differential 1 -form $\theta_{\nu, \mu}$ in (38), is that we can derive easily some invariance properties with respect to the group of rigid motions of the complex Hermitian space $\left(\mathbb{C}^{n}, d s^{2}\right)$; $d s^{2}=\sum_{j=1}^{n} d z_{j} \otimes d \overline{z_{j}}$. Thus, let $G$ denote the group of biholomorphic mapping of $\mathbb{C}^{n}$ that preserve the Hermitian metric $d s^{2}$. Then, $G=\mathbb{C}^{n} \rtimes U(n)$ is the group of semidirect product of the additive group $\left(\mathbb{C}^{n},+\right)$ with the unitary group $U(n)$ of $\mathbb{C}^{n}$ and can be represented as

$$
\begin{aligned}
G & :=\mathbb{C}^{n} \rtimes U(n) \\
& =\left\{g=\left(\begin{array}{ll}
A & b \\
0 & 1
\end{array}\right)=:[A, b] ; A \in U(n), b \in \mathbb{C}^{n}\right\} .
\end{aligned}
$$


It acts transitively on $\mathbb{C}^{n}$ via the mappings $g \cdot z=A z+b$. The pull-back $g^{*} \theta_{\nu, \mu}$ of the differential 1 -form $\theta_{\nu, \mu}$ by the above mapping $z \mapsto g$; $z$ is related to $\theta_{v, \mu}$ by the following identity.

Proposition 9. Let $\theta_{\nu, \mu}$ be as in (38). Then, for every $g \in G=$ $\mathbb{C}^{n} \rtimes U(n)$ we have

$$
g^{*} \theta_{\nu, \mu}=\theta_{\nu, \mu}+\frac{d j^{\nu, \mu}(g, z)}{j^{\nu, \mu}(g, z)},
$$

where

$$
j^{\nu, \mu}(g, z)=\exp \left(i \phi_{\nu, \mu}(g, z)\right) .
$$

The phase function $\phi_{\nu, \mu}(g, z)$ is given by

$$
\begin{aligned}
\phi_{\nu, \mu}(g, z)= & -v \operatorname{Re}\left(\left\langle z, g^{-1} \cdot 0\right\rangle\right) \\
& +\mu \Im m\left(\left\langle z, g^{-1} \cdot 0\right\rangle\right) .
\end{aligned}
$$

Proof. The identity (43) holds by component-wise straightforward computations. Indeed, direct computation yields

$$
\begin{aligned}
g^{*} \theta_{\nu, \mu}(z)= & \theta_{\nu, \mu}(z) \\
& -\frac{i \nu}{2} d\left[\left\langle z, g^{-1} \cdot 0\right\rangle+\overline{\left\langle z, g^{-1} \cdot 0\right\rangle}\right] \\
& +\frac{\mu}{2} d\left[\left\langle z, g^{-1} \cdot 0\right\rangle-\overline{\left\langle z, g^{-1} \cdot 0\right\rangle}\right] \\
= & \theta_{\nu, \mu}(z)+i d\left(\phi_{\nu, \mu}(g, z)\right),
\end{aligned}
$$

where $g^{-1}$ is the inverse mapping of $z \mapsto g \cdot z$ and $g^{-1} \cdot 0=$ $-A^{-1} b=-A^{*} b$ for $g=[A, b] \in \mathbb{C}^{n} \rtimes U(n)$. Thus, the result follows thanks to $d j^{\nu, \mu}(g, z)=i d\left(\phi_{\nu, \mu}(g, z)\right) j^{\nu, \mu}(g, z)$.

Notice that the relation (43) reads also as $g^{*} \theta_{\nu, \mu}=\theta_{\nu, \mu}+$ $d \log \left(j^{\nu, \mu}(\gamma, z)\right)$ and shows that the differential 1 -form $\theta_{\nu, \mu}$ is not $G$-invariant. But $g^{*} \theta_{\nu, \mu}$ and $\theta_{\nu, \mu}$ are in the same class of the de Rham cohomology group. Also it gives insight how to make, in view of the expression (39), the Laplacian $\Delta_{v, \mu}$ invariant with respect to a $G$-action on functions built with the help of the following automorphic factor $j^{\nu, \mu}(g, z)$ defined through (44) and satisfying the chain rule

$$
j^{\nu, \mu}\left(g g^{\prime}, z\right)=j^{\nu, \mu}\left(g, g^{\prime} z\right) j^{\nu, \mu}\left(g^{\prime}, z\right)
$$

for every $g, g^{\prime} \in G=\mathbb{C}^{n} \rtimes U(n)$ and $z \in \mathbb{C}^{n}$. Associated with $j^{\nu, \mu}$, we define $T_{g}^{\nu, \mu}$ to be the operator acting on differential $p$-forms $\omega$ of $\mathbb{C}^{n}$ through the formula

$$
T_{g}^{\nu, \mu} \omega=j^{\nu, \mu}(g, z) g^{*} \omega .
$$

On $\mathscr{C}^{\infty}$-complex-valued functions $f$ on $\mathbb{C}^{n}$, it reduces further to

$$
\begin{aligned}
{\left[T_{g}^{\nu, \mu} f\right](z) } & =j^{\nu, \mu}(g, z) g^{*} f(z) \\
& =j^{\nu, \mu}(g, z) f(g \cdot z) .
\end{aligned}
$$

Thus, the following invariance property for $\Delta_{\nu, \mu}$ holds.
Proposition 10. For every $g \in \mathbb{C}^{n} \rtimes U(n)$, we have

$$
\Delta_{\nu, \mu} T_{g}^{v, \mu}=T_{g}^{v, \mu} \Delta_{\nu, \mu}
$$

Proof. Using the well-known facts $g^{*} d=d g^{*}$ and $g^{*}(\alpha \wedge \beta)=$ $g^{*} \alpha \wedge g^{*} \beta$, we get

$$
\begin{aligned}
& T_{g}^{v, \mu}\left(\left(d+\operatorname{ext} \theta_{\nu, \mu}\right) f\right) \\
& \quad=j^{\nu, \mu}(\gamma, z)\left(d\left[g^{*} f\right]+\left[g^{*} \theta_{\nu, \mu}\right] \wedge\left[g^{*} f\right]\right) .
\end{aligned}
$$

Now, by means of the identity (43), it follows that

$$
\begin{aligned}
T_{g}^{v, \mu} & \left(\left(d+\operatorname{ext} \theta_{\nu, \mu}\right) f\right) \\
= & j^{\nu, \mu}(\gamma, z) d\left[g^{*} f\right]+j^{\nu, \mu}(\gamma, z) \theta_{\nu, \mu}\left[g^{*} f\right] \\
& \quad+d\left(j^{\nu, \mu}(\gamma, z)\right)\left[g^{*} f\right] \\
= & d\left(j^{\nu, \mu}(\gamma, z)\left[g^{*} f\right]\right)+\theta_{\nu, \mu} j^{\nu, \mu}(\gamma, z)\left[g^{*} f\right] \\
= & \left(d+\operatorname{ext} \theta_{\nu, \mu}\right)\left(T_{g}^{v, \mu} f\right) .
\end{aligned}
$$

Moreover, $T_{g}^{v, \mu}$ commutes also with $\left(d+\operatorname{ext} \theta_{\nu, \mu}\right)^{*}$ for $T_{g}^{\nu, \mu}$ being a unitary transformation. Therefore, by means of the expression of $\Delta_{\nu, \mu}=-\left(d+\operatorname{ext} \theta_{\nu, \mu}\right)^{*}\left(d+\operatorname{ext} \theta_{\nu, \mu}\right)$ as a magnetic Schrödinger operator $H_{\theta_{\nu, \mu}}$, we deduce easily that $\Delta_{\nu, \mu}$ and $T_{g}^{\nu, \mu}$ commute. This ends the proof.

Remark 11. For $g \in \mathbb{C}^{n} \rtimes\left\{I_{n}\right\}=\left(\mathbb{C}^{n},+\right)$, where $I_{n}$ denotes the identity matrix in $\mathbb{C}^{n \times n}$, the unitary operators $T_{g}^{\nu, \mu}$ given in (49) define projective representations of $G$ on the space of $\mathscr{C}^{\infty}$-functions on $\mathbb{C}^{n}$. In fact, they are the so-called magnetic translation operators that arise in the study of Schrödinger operators in the presence of uniform magnetic field.

Remark 12. The potential vector $\theta_{\nu, \mu}$ given through (38) can be seen as a specific magnetic vector potential that corresponds to an isotropic magnetic field of constant strength $2 \mu$, since $d\left(i \theta_{v, \mu}\right)(z)=2 \mu \operatorname{Vol}(z)$. It is issued from the holomorphic gauge $\theta_{\mu}^{h}$ and the symmetric gauge $\theta_{\mu}^{s}$ defined, respectively, by

$$
\begin{aligned}
& \theta_{\mu}^{h}=-\mu \sum_{j=1}^{n} \overline{z_{j}} d z_{j}, \\
& \theta_{\mu}^{s}=-\frac{\mu}{2} \sum_{j=1}^{n}\left(\overline{z_{j}} d z_{j}-z_{j} d \overline{z_{j}}\right) .
\end{aligned}
$$

More exactly, we have the gauge transformations

$$
\begin{aligned}
& \theta_{\nu, \mu}=\theta_{\mu}^{h}+\frac{i \nu+\mu}{2} d \sum_{j=1}^{n}\left|z_{j}\right|^{2}, \\
& \theta_{\nu, \mu}=\theta_{\mu}^{s}+\frac{i \nu}{2} d \sum_{j=1}\left|z_{j}\right|^{2} .
\end{aligned}
$$


Remark 13. The connection form $\theta_{\nu, \mu}$ in (38) reads

$$
\theta_{v, \mu}(z)=i \sum_{j=1}^{n}\left(\mu y_{j}+v x_{j}\right) d x_{j}-\left(\mu x_{j}-v y_{j}\right) d y_{j}
$$

in the real coordinates $\left(x_{1}, y_{1}, x_{2}, y_{2}, \ldots, x_{n} ; y_{n}\right)$. Its divergence is then showed to be given by $\operatorname{div} \theta_{\nu, \mu}=2 i n \nu$. Accordingly, $\operatorname{div} \theta_{\nu, \mu}$ corresponds to the constant term involved in the expression of the magnetic quantum Hamiltonian $\Delta_{\nu, \mu}$ given through (34). Therefore, $\theta_{\nu, \mu}$ is not a radiation (coulomb) gauge, unless $\nu=0$, and therefore $\theta_{\nu, \mu}$ can be seen as a perturbation of the Coulomb gauge. The perturbation operator in $\Delta_{\nu, \mu}$ is given by

$$
D_{v}:=2 i v(E+\bar{E}+n)-v^{2}|z|^{2},
$$

so that $\Delta_{\nu, \mu}=H_{\mu}+D_{\nu}$.

\section{Spectral Properties of $\Delta_{\nu, \mu}$ Acting on}

$$
\mathscr{C}^{\infty}\left(\mathbb{C}^{n}\right) \text { and on } \mathscr{H}=L^{2}\left(\mathbb{C}^{n}, d m\right)
$$

We denote by $\mathscr{C}^{\infty}\left(\mathbb{C}^{n}\right)$ the Frechet space of complex-valued functions on $\mathbb{C}^{n}$ endowed with the compact-open topology, while $L^{2}\left(\mathbb{C}^{n}, d m\right)$ denotes the usual Hilbert space of square integrable complex-valued functions $F(z)$ on $\mathbb{C}^{n}$ with respect to the usual Lebesgue measure $d m$. In the sequel, we will give a concrete description of the eigenspaces of $\Delta_{\nu, \mu}$ in both $\mathscr{C}^{\infty}\left(\mathbb{C}^{n}\right)$ and $L^{2}\left(\mathbb{C}^{n}, d m\right)$. To this end, let $\lambda$ be any complex number and denote by $E_{\lambda}\left(\Delta_{\gamma, \mu}\right)$ the eigenspace of $\Delta_{\nu, \mu}$ corresponding to the eigenvalue $-2 \mu(2 \lambda+n)$ in $\mathscr{C}^{\infty}\left(\mathbb{C}^{n}\right)$; that is,

$$
\begin{aligned}
E_{\lambda} & \left(\Delta_{\nu, \mu}\right) \\
& =\left\{F \in \mathscr{C}^{\infty}\left(\mathbb{C}^{n}\right) ; \Delta_{\nu, \mu} F=-2 \mu(2 \lambda+n) F\right\} .
\end{aligned}
$$

Also, by $\mathscr{F}_{\lambda}^{2}\left(\Delta_{v, \mu}\right)$ we denote the subspace of $L^{2}\left(\mathbb{C}^{n}, d m\right)$ whose elements satisfy $\Delta_{\nu, \mu} F=-2 \mu(2 \lambda+n) F$. Namely, by elliptic regularity of $\Delta_{\nu, \mu}$, we have

$$
\mathscr{F}_{\lambda}^{2}\left(\Delta_{\gamma, \mu}\right):=L^{2}\left(\mathbb{C}^{n}, d m\right) \cap E_{\lambda}\left(\Delta_{\nu, \mu}\right)
$$

The first result related to $E_{\lambda}\left(\Delta_{\nu, \mu}\right)$ and $\mathscr{F}_{\lambda}^{2}\left(\Delta_{\nu, \mu}\right)$ is the following.

Proposition 14. The eigenspaces $E_{\lambda}\left(\Delta_{\nu, \mu}\right)$ and $\mathscr{F}_{\lambda}^{2}\left(\Delta_{\nu, \mu}\right)$ are invariants under the $T^{\nu, \mu}$-action given by (49), in the sense that for every $g \in G=\mathbb{C}^{n} \rtimes U(n)$ we have

$$
\begin{gathered}
T_{g}^{\nu, \mu}\left(E_{\lambda}\left(\Delta_{\nu, \mu}\right)\right) \subset E_{\lambda}\left(\Delta_{\nu, \mu}\right), \\
T_{g}^{\nu, \mu}\left(\mathscr{F}_{\lambda}^{2}\left(\Delta_{\nu, \mu}\right)\right) \subset \mathscr{F}_{\lambda}^{2}\left(\Delta_{\nu, \mu}\right) .
\end{gathered}
$$

Proof. This can be handled easily making use of the invariance property (50) of $\Delta_{\gamma, \mu}$ by the unitary transformations $T_{g}^{\nu, \mu}$
Proposition 15. The set of spherical eigenfuctions of $\Delta_{\nu, \mu}$ with $-2 \mu(2 \lambda+n)$ as eigenvalue is a one-dimensional vector subspace of $E_{\lambda}\left(\Delta_{\gamma, \mu}\right)$ generated by

$$
\varphi_{\lambda}^{\nu, \mu}(z)=e^{-((\mu-i v) / 2)|z|^{2}}{ }_{1} F_{1}\left(-\lambda ; n ; \mu|z|^{2}\right),
$$

where ${ }_{1} F_{1}(a ; c ; x)$ is denoting here the usual confluent hypergeometric function

$$
{ }_{1} F_{1}(a ; c ; x)=1+\frac{a}{c} \frac{x}{1 !}+\frac{a(a+1)}{c(c+1)} \frac{x^{2}}{2 !}+\cdots ;
$$

$x \in \mathbb{C}$.

Remark 16. By a "spherical" (or radial here) eigenfuction of $\Delta_{v, \mu}$, we mean any $U(n)$-invariant function $f$ satisfying $f(h$. $z)=f(z)$ for all $h \in U(n)$ and $z \in \mathbb{C}^{n}$.

Proof (sketched). To prove the statement, we write $\Delta_{\nu, \mu}$ in the polar coordinates $z=r \theta$ with $r \geq 0$ and $\theta \in S^{2 n-1}$ as

$$
\begin{aligned}
\Delta_{\nu, \mu}= & \frac{\partial^{2}}{\partial r^{2}}+\left(\frac{2 n-1}{r}+2 i \nu\right) \frac{\partial}{\partial r}-\left(\nu^{2}+\mu^{2}\right) r^{2} \\
& +2 i v n+L_{\nu, \mu}^{\theta},
\end{aligned}
$$

where $L_{\nu, \mu}^{\theta}$ stands for the tangential component of $\Delta_{\nu, \mu}$. The eigenvalue problem $\Delta_{\nu, \mu} f=-2 \mu(2 \lambda+n) f$ for radial functions $f(z)=\psi(x)$, with $x=r^{2}$ reduces to the differential equation

$$
\begin{aligned}
& \left\{x \frac{\partial^{2}}{\partial x^{2}}+(n+i v x) \frac{\partial}{\partial x}\right. \\
& \left.-\left[\frac{\nu^{2}+\mu^{2}}{4} x+\frac{i \nu-\mu}{2} n-\mu \lambda\right]\right\} \psi=0 .
\end{aligned}
$$

Next, making use of the appropriate change of function $\psi(x)=e^{((i v-\mu) / 2) x} y(x)$, we see that the previous equation leads to the confluent hypergeometric differential equation [20, page 193]

$$
x y^{\prime \prime}+(n-\mu x) y^{\prime}+\mu \lambda y=0
$$

whose regular solution at $x=0$ is the confluent hypergeometric function ${ }_{1} F_{1}(-\lambda ; n ; \mu x)$.

Remark 17. The existence of the gradient of the function $\varphi(z)=(i \nu / 2)|z|^{2}$, in the gauge transformation $\theta_{\nu, \mu}=\theta_{\mu}^{s}+$ $(i \nu / 2) d|z|^{2}$, is equivalent to multiplying the eigenstates of the Landau Hamiltonian $H_{\mu} ; v=0$, by the phase factor $e^{-(i v / 2)|z|^{2}}$. In fact, this follows by considering the similarity transformation generated by the unitary operator $S f=$ $e^{-(i v / 2)|z|^{2}} f$ and next showing that

$$
\Delta_{\nu, \mu}=e^{-(i v / 2)|z|^{2}} \Delta_{0, \mu} e^{+(i v / 2)|z|^{2}} .
$$

This is clear from the proof of Proposition 15. In other words the operators $\Delta_{\nu, \mu}$ and $\Delta_{0, \mu}$ are unitary equivalent in $L^{2}\left(\mathbb{C}^{n}, d m\right)$. 
Remark 18. According to Remark 17, any physical interpretation or application must take into account the parameter $v$ that is related to the special gauge transformation we have made.

Remark 19. The key observation is contained in the identity (65) which will serve as an outline of the proof of Proposition 15 as well as the proofs of the assertions below, taking into account the well-established results for $\Delta_{0, \mu}$ (see $[1,4-7]$ and the references therein).

Accordingly, we claim the following.

Proposition 20. Let $(\nu, \mu) \in \mathbb{R}^{2}$ with $\mu>0$ and $\lambda \in \mathbb{C}$. Then, the eigenspace $\mathscr{F}_{\lambda}^{2}\left(\Delta_{v, \mu}\right)$ as defined in (58) is nonzero (Hilbert) space if and only if $\lambda=l$ with $l=0,1,2, \ldots$, is a positive integer number. Moreover, the spaces $\mathscr{F}_{l}^{2}\left(\Delta_{v, \mu}\right), l=0,1,2, \ldots$, are pairwise orthogonal in $L^{2}\left(\mathbb{C}^{n}, d m\right)$ and we have the following orthogonal decomposition in Hilbertian subspaces:

$$
L^{2}\left(\mathbb{C}^{n}, d m\right)=\bigoplus_{l=0}^{\infty} \mathscr{F}_{l}^{2}\left(\Delta_{\nu, \mu}\right) .
$$

Remark 21. A direct proof of Proposition 20 can be handled using Proposition 15 and the asymptotic behavior of the confluent hypergeometric function given by [20, page 332]

$$
\begin{aligned}
& { }_{1} F_{1}(a ; c ; x) \\
& \quad=\Gamma(c)\left\{\frac{(-x)^{-a}}{\Gamma(c-a)}+\frac{e^{x} x^{a-c}}{\Gamma(a)}\right\}\left(1+O\left(\frac{1}{x}\right)\right)
\end{aligned}
$$

as $x \rightarrow+\infty$. This asymptotic behavior can also be used to show that the radial function $\varphi_{\lambda}^{\nu, \mu}$ given by (60) is bounded if and only if $\lambda=l ; l=0,1,2, \ldots$.

Remark 22. The unitary equivalence of $\Delta_{\nu, \mu}$ and $\Delta_{0, \mu}$ in $L^{2}\left(\mathbb{C}^{n}, d m\right)$ shows that the spectrum of $\Delta_{\nu, \mu}$ is purely discrete and coincides with the one of $\Delta_{0, \mu}$. Thus, the energy levels (eigenvalues) are independent of the $\nu$-parameter and are quantized as $-2 \mu(2 l+n)$ for varying $l \in \mathbb{Z}^{+}$. This is also contained in Proposition 20. Moreover, each energy level is infinitely degenerate, since it corresponds to infinite linearly independent states of the quantum system. The states $\psi_{p, q \mid l}$, given by (68) below, with index $l$ constitute the $l$ th Landau level.

The following result prescribes the elements of the $L^{2}$ eigenspaces of $\Delta_{v, \mu}$ in terms of the confluent hypergeometric functions ${ }_{1} F_{1}$ and the harmonic polynomials $h^{p q}(z, \bar{z})$ on $\mathbb{C}^{n}$ that are homogeneous of degree $p$ in $z$ and degree $q$ in $\bar{z}$ (see [21]).

Proposition 23. An orthogonal basis of the infinite dimensional eigengspace $\mathscr{F}_{l}^{2}\left(\Delta_{\nu, \mu}\right)$ in (58) is given by

$$
\begin{aligned}
& \psi_{p, q \mid l}^{\mu}(z)=e^{-((\mu+i v) / 2)|z|^{2}}{ }_{1} F_{1}\left(q-l, n+p+q ; \mu|z|^{2}\right) \\
& \cdot h^{p q}(z, \bar{z}),
\end{aligned}
$$

for arbitrary nonnegative integers $p, q$ with $q \leq l$. Moreover, a function $F$ belongs to $\mathscr{F}_{l}^{2}\left(\Delta_{\nu, \mu}\right)$ if and only if it can be expanded as follows:

$$
F(z)=\sum_{p=0}^{+\infty} \sum_{q=0}^{l} a_{p, q} \psi_{p, q \mid l}^{\mu}(z),
$$

where the constants $a_{p, q}$ satisfy the growth condition

$$
\begin{aligned}
\|F\|_{L^{2}\left(\mathbb{C}^{n} ; d m\right)}^{2} & =\sum_{p=0}^{+\infty} \sum_{q=0}^{l}\left|a_{p, q}\right|^{2}\left(\frac{1}{\mu}\right)^{n+p+q} \\
& \frac{(l-q) ! \Gamma^{2}(n+p+q)}{2 \Gamma(n+p+l)}\left\|h^{p q}\right\|_{L^{2}\left(S^{2 n-1}\right)}^{2}<+\infty .
\end{aligned}
$$

Proof. The result follows immediately from the similarity transformation (65) combined with (ii) in Proposition 6 in $[5]$.

We conclude this section with the following assertion.

Proposition 24. Let $(\nu, \mu) \in \mathbb{R}^{2}$ with $\mu>0$. For fixed $l=$ $0,1,2, \ldots$, let $P_{l}$ be the orthogonal eigenprojector operator from $L^{2}\left(\mathbb{C}^{n}, d m\right)$ onto the eigenspace $\mathscr{F}_{l}^{2}\left(\Delta_{\nu, \mu}\right)$ with $-2 \mu(2 l+n)$ as eigenvalue. Then the Schwartz kernel $P_{l}^{\nu, \mu}(z, w)$ of the operator $P_{l}$ is given by the following explicit formula:

$$
\begin{aligned}
P_{l}^{\nu, \mu}(z, w)= & \left(\frac{\mu}{\pi}\right)^{n} \frac{(n-1+l) !}{(n-1) ! l !} j^{\nu, \mu}(z, w) \\
& \cdot e^{-(\mu / 2)|z-w|^{2}}{ }_{1} F_{1}\left(-l ; n ; \mu|z-w|^{2}\right),
\end{aligned}
$$

where the factor $j^{\nu, \mu}(z, w), z, w \in \mathbb{C}^{n}$, is given by

$$
j^{\nu, \mu}(z, w)=e^{-(i v / 2)\left(|z|^{2}-|w|^{2}\right)+(\mu / 2)(\langle z, w\rangle-\overline{\langle z, w\rangle})} .
$$

Proof (sketched). The proof for $v=0$ is contained in [1, 4, 5]. For arbitrary $v$, the proof can be handled in a similar way or making use of the key observation that in $L^{2}\left(\mathbb{C}^{n}, d m\right)$, the operators $\Delta_{\nu, \mu}$ and $\Delta_{0, \mu}$ are unitary equivalents and we have

$$
\Delta_{\nu, \mu}=e^{-(i v / 2)|z|^{2}} \Delta_{0, \mu} e^{+(i v / 2)|z|^{2}} .
$$

\section{Factorization of $\Delta_{\gamma, \mu}$ and Associated Hermite Polynomials}

In this section we study the spectral theory of $\Delta_{\nu, \mu}$ on $L^{2}\left(\mathbb{C}^{n}, d \lambda\right)$ using the factorization method. This method finds its origin in the works of Dirac [12] and Schrödinger [13], then developed by Infeld and Hull [14] in order to solve eigenvalue problems appearing in quantum theory. For a recent survey see [16]. The key observation is that the Laplacian $\Delta_{\nu, \mu}$ can be rewritten as

$$
\begin{aligned}
& \Delta_{\nu, \mu}=-4 \sum_{j=1}^{n}\left(-\frac{\partial}{\partial z_{j}}+\frac{\mu-i \nu}{2} \overline{z_{j}}\right)\left(\frac{\partial}{\partial \overline{z_{j}}}+\frac{\mu+i \nu}{2} z_{j}\right) \\
& \quad-2 \mu n I .
\end{aligned}
$$


The spectral properties of $\Delta_{\gamma, \mu}$ on $L^{2}\left(\mathbb{C}^{n}, d m\right)$ or on $\mathscr{C}^{\infty}\left(\mathbb{C}^{n}\right)$, discussed in the previous section, can be derived from Landau's work (see [22]). In the sequel, we will adopt the operator

$$
\widetilde{\Delta}_{\nu, \mu}:=-\frac{1}{4} \Delta_{\nu, \mu} .
$$

The extra factor $-1 / 4$ is present to simplify later formulas. According to factorization above, we defined for every $j=$ $1,2, \ldots, n$, the following first-order differential operators

$$
\begin{aligned}
& a_{j}^{+}=-\frac{\partial}{\partial z_{j}}+\frac{\mu-i v}{2} \overline{z_{j}}=-e^{((\mu-i v) / 2)\left|z_{j}\right|^{2}} \frac{\partial}{\partial z_{j}} e^{-((\mu-i v) / 2)\left|z_{j}\right|^{2}}, \\
& a_{j}^{-}=\frac{\partial}{\partial \overline{z_{j}}}+\frac{\mu+i v}{2} z_{j}=e^{-((\mu+i v) / 2)\left|z_{j}\right|^{2}} \frac{\partial}{\partial \overline{z_{j}}} e^{((\mu+i v) / 2)\left|z_{j}\right|^{2}} .
\end{aligned}
$$

These operators satisfy the commutation relationships $\left[a_{j}^{-}, a_{k}^{+}\right]=n \mu \delta_{j, k}$, where $\delta_{j, k}$ is the Krönecker symbol. They are linked to the Laplacian $\widetilde{\Delta}_{\gamma, \mu}$ through

$$
\begin{aligned}
& \sum_{j=1}^{n} a_{j}^{+} a_{j}^{-}=\widetilde{\Delta}_{\gamma, \mu}-\frac{n}{2} \mu, \\
& \sum_{j=1}^{n} a_{j}^{-} a_{j}^{+}=\widetilde{\Delta}_{\gamma, \mu}+\frac{n}{2} \mu .
\end{aligned}
$$

Moreover, we have the following creation and annihilation identities:

$$
\begin{aligned}
& \widetilde{\Delta}_{\gamma, \mu} a_{j}^{+}=a_{j}^{+}\left(\widetilde{\Delta}_{\gamma, \mu}+\mu\right), \\
& \widetilde{\Delta}_{\gamma, \mu} a_{j}^{-}=a_{j}^{-}\left(\widetilde{\Delta}_{\gamma, \mu}-\mu\right)
\end{aligned}
$$

and allow the determination of the eigenvalues and eigenvectors of $\widetilde{\Delta}_{\gamma, \mu}$. Indeed, if $\psi$ is an eigenvector of $\widetilde{\Delta}_{\gamma, \mu}$ associated with the eigenvalue $\lambda$ we have the following:

$$
\begin{aligned}
& \widetilde{\Delta}_{v, \mu}\left(a_{j}^{+} \psi\right)=a_{j}^{+}\left(\widetilde{\Delta}_{\nu, \mu}+\mu\right) \psi=(\lambda+\mu) a_{j}^{+} \psi, \\
& \widetilde{\Delta}_{\nu, \mu}\left(a_{j}^{-} \psi\right)=a_{j}^{-}\left(\widetilde{\Delta}_{v, \mu}-\mu\right) \psi=(\lambda-\mu) a_{j}^{-} \psi .
\end{aligned}
$$

Thus, we need only to know those associated with the lowest eigenvalue. In fact, since $\widetilde{\Delta}_{\nu, \mu}$ is positive semidefinite, all the eigenvalues are real and nonnegative. Moreover, from symmetry and ellipticity of $\widetilde{\Delta}_{\gamma, \mu}$ we know that $\widetilde{\Delta}_{\gamma, \mu}$ has an infinite sequence of nonnegative eigenvalues (see, e.g., [10]):

$$
0 \leq \lambda_{0}<\lambda_{1}<\cdots \uparrow \infty .
$$

Therefore, if $\psi_{0}$ is an eigensolution associated with $\lambda_{0}$, we have necessary $a_{j}^{-} \psi_{0}=0$ for every $j=1,2, \ldots, n$, thanks to (81) and therefore $\psi_{0}(z)=e^{-((\mu+i v) / 2)|z|^{2}} f(z)$, where $f$ is any arbitrary holomorphic function. This follows readily making use the expression of $a_{j}^{-}$in (77) involving the exponential. Consequently,

$$
\begin{aligned}
\mathbf{A}_{0} & =\operatorname{ker}\left(a_{j}^{-}\right) \\
& =\overline{\operatorname{span}}\left\{z^{m} e^{-((\mu+i v) / 2)|z|^{2}} ; m \in\left(\mathbb{Z}^{+}\right)^{n}\right\},
\end{aligned}
$$

where $z^{m}$ for given multi-index $m:=\left(m_{1}, m_{2}, \ldots, m_{n}\right)$ means $z^{m}:=z_{1}^{m_{1}} z_{2}^{m_{2}} \cdots z_{n}^{m_{n}}$. Making use of the creation operators we obtain the following family of multi-indexed functions $h_{r, s}^{\nu, \mu}$; $r=\left(r_{1}, \ldots, r_{n}\right) ; s=\left(s_{1}, \ldots, s_{n}\right)$ :

$$
\begin{aligned}
h_{r, s}^{\gamma, \mu} & =\left(a_{1}^{+}\right)^{r_{1}} \cdots\left(a_{n}^{+}\right)^{r_{n}}\left(z^{s} e^{-((\mu+i v) / 2)|z|^{2}}\right)=(-1)^{|r|} \\
\cdot & e^{((\mu-i v) / 2)|z|^{2}} D_{z}^{r} e^{-((\mu-i v) / 2)|z|^{2}}\left(z^{s} e^{-((\mu+i v) / 2)|z|^{2}}\right) \\
& =(-1)^{|r|+|s|} \mu^{-|s|} e^{((\mu-i v) / 2)|z|^{2}} D_{z}^{r} D_{\bar{z}}^{s} e^{-\mu|z|^{2}}
\end{aligned}
$$

where $|m|$ and $m$ ! stand for $|m|:=m_{1}+\cdots+m_{2}$ and $m !:=$ $m_{1} ! \cdots m_{n} !$, respectively, and $D_{z}^{m}$ and $D_{\bar{z}}^{m}$ are defined by

$$
\begin{aligned}
& D_{z}^{m}:=\frac{\partial^{|m|}}{\partial z_{1}^{m_{1}} \cdots \partial z_{n}^{m_{n}}}, \\
& D_{\bar{z}}^{m}:=\frac{\partial^{|m|}}{\partial \bar{z}_{1}^{m_{1}} \cdots \partial \bar{z}_{n}^{m_{n}}} .
\end{aligned}
$$

According to the above discussion, $h_{r, s}^{v, \mu}$ are eigensolutions associated with the eigenvalue $\lambda_{l}:=\mu(n / 2+l) ; l=|r|=$ $0,1,2, \ldots$, of $\widetilde{\Delta}_{v, \mu}$. The following proposition shows that

$$
\lambda_{l}:=\mu\left(\frac{n}{2}+l\right) ; \quad l=0,1,2, \ldots
$$

are the only eigenvalues of $\widetilde{\Delta}_{v, \mu}$.

Proposition 25. $h_{r, s}^{\nu, \mu}$ form a complete orthogonal system in the Hilbert space $L^{2}\left(\mathbb{C}^{n}, d \lambda\right)$. Moreover, we have the following decomposition $L^{2}\left(\mathbb{C}^{n}, d \lambda\right)=\bigoplus_{l=0}^{\infty} \mathbf{A}_{l}$, where

$$
\mathbf{A}_{l}:=\overline{\operatorname{span}}\left\{h_{r, s}^{v, \mu} ; r, s \in\left(\mathbb{Z}^{+}\right)^{n},|r|=l\right\} .
$$

Proof. The identity (84) shows that $h_{r, s}^{\nu, \mu}$, up to $e^{i v|z|^{2} / 2}$, are essentially the high-dimensional analogue of the univariate complex Hermite functions

$$
\begin{aligned}
h_{m, n}^{\sigma}(\xi, \bar{\xi}):=(-1)^{m+n} e^{\sigma|\xi|^{2}} \frac{\partial^{m+n}}{\partial \xi^{m} \partial \bar{\xi}^{n}} e^{-\sigma|\xi|^{2}} ; & \\
\xi & \in \mathbb{C}, \sigma>0,
\end{aligned}
$$

considered in $[23,24]$. The main idea of the proof is then to separate the variable $z$ in the expression (84) into its components $z_{j}$ to get

$$
\begin{aligned}
& h_{r, s}^{\nu, \mu}(z, \bar{z}) \\
& =\mu^{-|s|} e^{((-\mu-i v) / 2)|z|^{2}} \prod_{j=1}^{n}(-1)^{r_{j}+s_{j}} e^{\mu\left|z_{j}\right|^{2}} \frac{\partial^{r_{j}+s_{j}}}{\partial z_{j}^{r_{j}} \partial \bar{z}_{j}^{s_{j}}} e^{-\mu\left|z_{j}\right|^{2}} \\
& =\mu^{-|s|} e^{((-\mu-i v) / 2)|z|^{2}} \prod_{j=1}^{n} h_{r_{j}, s_{j}}\left(\sqrt{\mu} z_{j}, \sqrt{\mu \bar{z}_{j}}\right) .
\end{aligned}
$$


This implies that the eigenvalues of $\Delta_{\gamma, \mu}=-4 \widetilde{\Delta}_{\nu, \mu}$ are

$$
\{-2 \mu(n+2 l) ; l=0,1,2, \ldots\},
$$

which coincide with the results in Section 5. Notice as well that $\mathscr{F}_{l}^{2}\left(\Delta_{v, \mu}\right)$ and $\mathbf{A}_{l}$ refer to the same set.

Remark 26. The Hermite functions $h_{r, s}^{\nu, \mu}$ are given explicitly by

$$
\begin{aligned}
& h_{r, s}^{\nu, \mu} \\
& =\mu^{-|s|} e^{((-\mu-i v) / 2)|z|^{2}} \sum_{|k|=0}^{\min (r, s)} \frac{\left(\sqrt{\left.\mu^{|r|+|| s|-2| k \mid}\right)(-1)^{|r-s|} r ! s !}\right.}{k !(r-k) !(s-k) !} z^{s-k} \bar{z}^{r-k},
\end{aligned}
$$

where $r=\left(r_{1}, \ldots, r_{n}\right), s=\left(s 1, \ldots, s_{n}\right)$, and $\min (r, s):=$ $\left(\min \left(r_{1}, s_{1}\right), \ldots, \min \left(r_{n}, s_{n}\right)\right)$.

\section{Concluding Remarks}

The consideration of the unitary transformations $T_{g}^{v, \mu}, g \in$ $G=\mathbb{C}^{n} \rtimes U(n)$, and the $T_{g}^{\nu, \mu}$-invariance property satisfied by the magnetic Laplacian $\Delta_{\nu, \mu}$ give rise to new class of biweighted automorphic functions associated with the automorphic factor $j^{\nu, \mu}(g, z)$ when we restrict $g$ to belong in a full-rank discrete subgroup $\Gamma$ of $G$. We call them $\Gamma$ periodic functions of biweight $(\nu, \mu)$. The considered $\Delta_{v, \mu}$ leaves invariant this space and therefore the corresponding eigenvalue problem is well-defined. Thus, a detailed description of the spectral properties of $\Delta_{\gamma, \mu}$ when acting on biweighted automorphic functions with respect to any discrete subgroup of $\left(\mathbb{C}^{n},+\right)$ (not necessary of full-rank) is of great interest. In this context, the particular case $v=0$ and $\Gamma=\mathbb{C}^{n} \rtimes\{1\}$, these functions reduce further the classical one studied in [11]. We hope to focus on this in a near future.

\section{Conflicts of Interest}

The authors declare that there are no conflicts of interest regarding the publication of this article and regarding the funding that they have received.

\section{Acknowledgments}

The present investigation was completed during the secondnamed author's visit to Dipartimento di Matematica of Politecnico di Milano May-June 2017. He would like to express his gratitude to Professors F. Colombo and I. M. Sabadini for hospitality. The authors are partially supported by the Hassan II Academy of Sciences and Technology, Morocco. This research work was partially supported by a grant from the Simons Foundation to Allal Ghanmi.

\section{References}

[1] J. Avron, I. Herbst, and B. Simon, "Schrödinger operators with magnetic fields. I. General interactions," Duke Mathematical Journal, vol. 45, no. 4, pp. 847-883, 1978.

[2] S. Thangavelu, "Lectures on Hermite and Laguerre expansions," vol. 42 of Mathematical Notes, Princeton University Press, Princeton, NJ, USA, 1993.
[3] M. W. Wong, The Weyl transform, Springer, New York, NY, USA, 1998.

[4] N. E. Askour, A. Intissar, and Z. r. Mouayn, "Explicit formulas for reproducing kernels of generalized Bargmann spaces on $\mathbb{C}^{n}$," Journal of Mathematical Physics, vol. 41, no. 5, pp. 3057-3067, 2000.

[5] A. Ghanmi and A. Intissar, "Asymptotic of complex hyperbolic geometry and $\mathrm{L}^{2}$-spectral analysis of Landau-like hamiltonians," Journal of Mathematical Physics, vol. 46, no. 3, Article ID 032107, 032107, 26 pages, 2005.

[6] J. Tie, "The twisted Laplacian on $\mathbb{C}^{n}$ and the sub-Laplacian on $\mathrm{H}_{n}$," Communications in Partial Differential Equations, vol. 31, no. 7-9, pp. 1047-1069, 2006.

[7] H. Koch and F. Ricci, "Spectral projections for the twisted Laplacian," Studia Mathematica, vol. 180, no. 2, pp. 103-110, 2007.

[8] J. Bellissard, "Number Theory to Physics," in Number Theory to Physics, M. Waldschmidt, Ed., pp. 538-630, Springer-Verlag, Berlin, Germany, 2nd edition, 1992.

[9] J. Asch, H. Over, and R. Seiler, "Magnetic Bloch analysis and Bochner Laplacians," Journal of Geometry and Physics, vol. 13, no. 3, pp. 275-288, 1994.

[10] R. Kuwabara, "On spectra of the Laplacian on vector bundles," Journal of Mathematics. Tokushima University, vol. 16, pp. 1-23, 1982.

[11] A. Ghanmi and A. Intissar, "Landau automorphic functions on $\mathbb{C}^{n} v$," Journal of Mathematical Physics, vol. 49, no. 8, Article ID 083503, 083503, 20 pages, 2008.

[12] P. A. M. Dirac, The principles of quantum mechanics. Number 2, Oxford university press, Oxford, UK, 4th edition, 1935.

[13] E. Schrödinger, "A method of determining quantummechanical eigenvalues and eigenfunctions," Proceedings of the Royal Irish Academy. Section A: Mathematical and Physical Sciences, vol. 46, pp. 9-16, 1940.

[14] L. Infeld and T. E. Hull, “The factorization method," Reviews of Modern Physics, vol. 23, pp. 21-68, 1951.

[15] B. Mielnik, "Factorization method and new potentials with the oscillator spectrum," Journal of Mathematical Physics, vol. 25, no. 12 , pp. 3387-3389, 1984.

[16] S.-H. Dong, "Factorization method in quantum mechanics," in Fundamental Theories of Physics, vol. 150, Springer, Dordrecht, The Netherlands, 2007.

[17] G. B. Folland, Harmonic analysis in phase space, vol. 122 of Annals of Mathematics Studies, Princeton University Press, Princeton, NJ, USA, 1989.

[18] Y. I. Manin, "Quantized theta-functions," Progress of theoretical physics. Supplement, no. 102, pp. 219-228, 1991.

[19] M. Shubin, "Essential self-adjointness for semi-bounded magnetic Schrödinger operators on non-compact manifolds," Journal of Functional Analysis, vol. 186, no. 1, pp. 92-116, 2001.

[20] A. Nikiforov and V. Uvarov, Fonctions spéciales de la physique mathématique, Mir, Moscow, Russia, 1978.

[21] G. B. Folland, "Spherical harmonic expansion of the PoissonSzegö kernel for the ball," Proceedings of the American Mathematical Society, vol. 47, no. 2, pp. 401-408, 1975.

[22] L. Landau and E. Lifshitz, Quantum Mechanics: Non-relativistic Theory, Butterworth-Heinemann, Oxford, UK, 1977.

[23] K. Itô, "Complex multiple Wiener integral," Japanese Journal of Mathematics, vol. 22, pp. 63-86, 1952.

[24] A. Ghanmi, "A class of generalized complex Hermite polynomials," Journal of Mathematical Analysis and Applications, vol. 340, no. 2, pp. 1395-1406, 2008. 


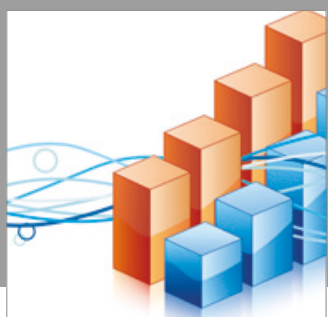

Advances in

Operations Research

vatersals

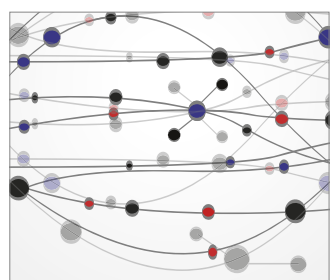

\section{The Scientific} World Journal
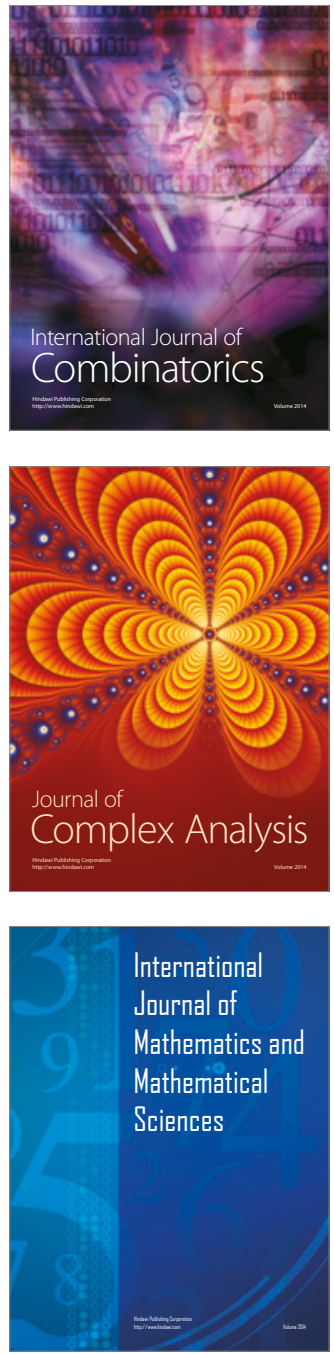
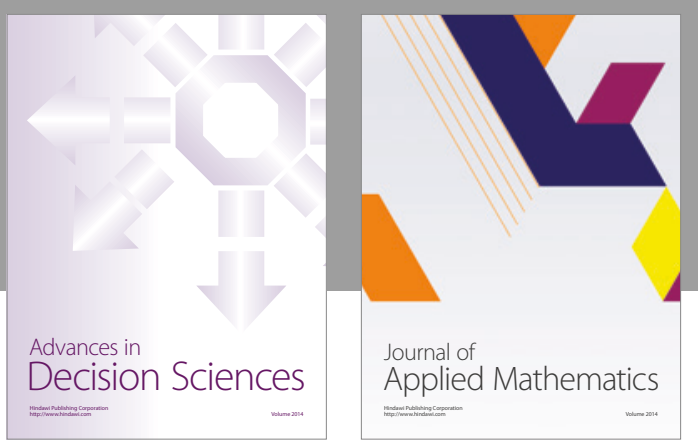

Algebra

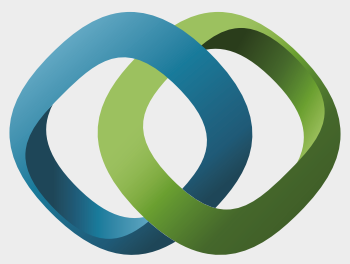

\section{Hindawi}

Submit your manuscripts at

https://www.hindawi.com
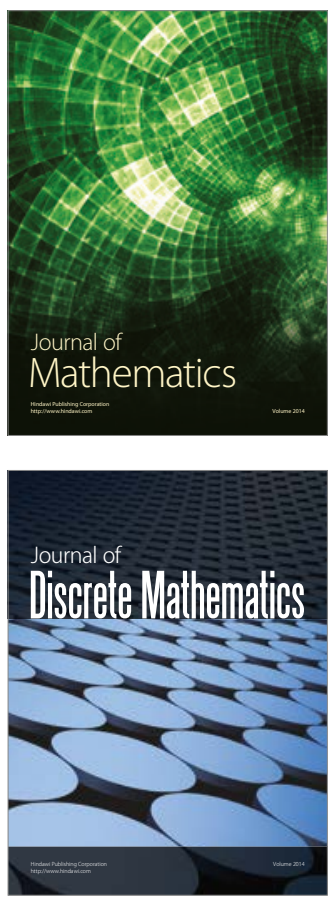

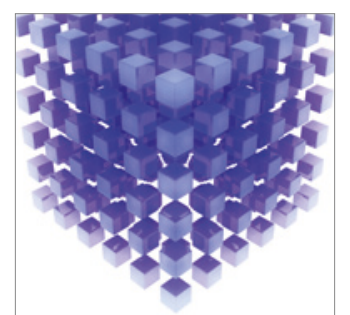

Mathematical Problems in Engineering
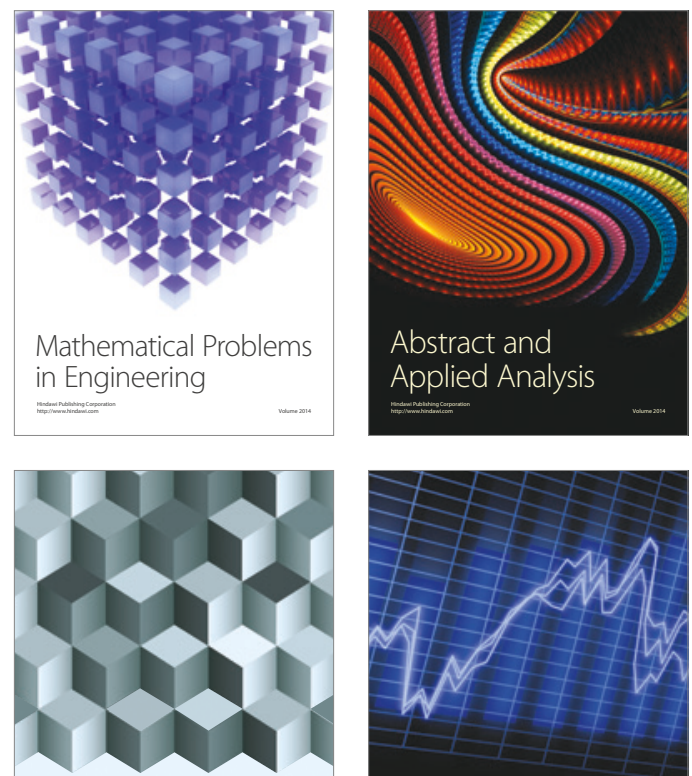

Journal of

Function Spaces

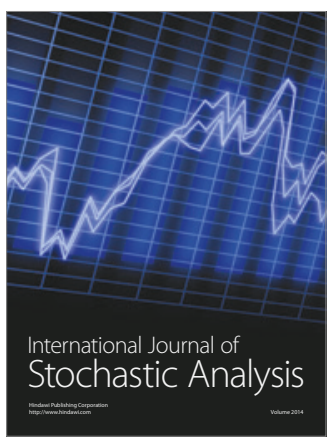

Probability and Statistics
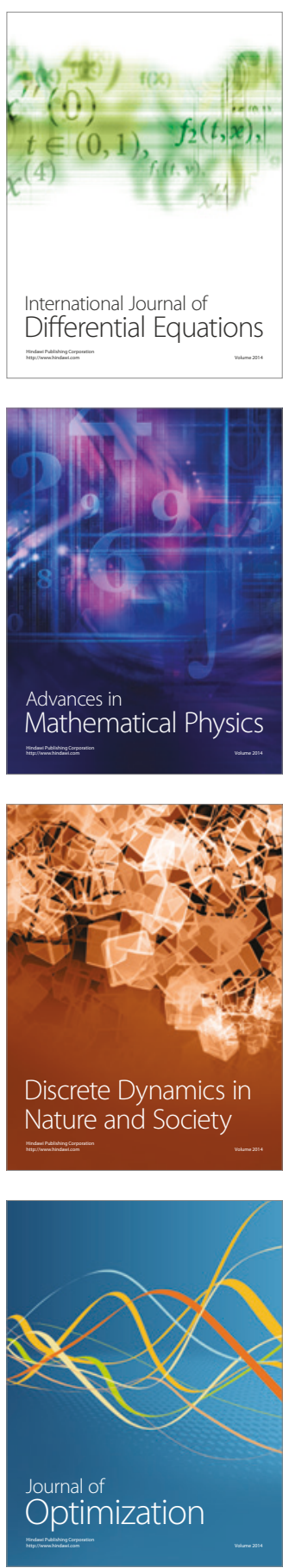